\title{
Impact of hemostatic methods on ovarian reserve and fertility in laparoscopic ovarian cystectomy
}

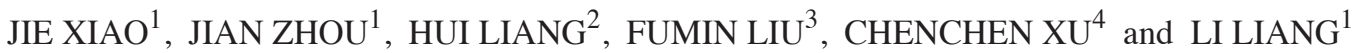 \\ ${ }^{1}$ Department of Gynecology and ${ }^{2}$ Cervical Disease Center, Xuzhou Maternity and Child Health Care Hospital, \\ Xuzhou Medical University; ${ }^{3}$ Department of Gynaecology and Obstetrics, The Affiliated Hospital \\ of Xuzhou Medical University; ${ }^{4}$ B Ultrasonic Room, Xuzhou Maternity and Child Health \\ Care Hospital, Xuzhou Medical University, Xuzhou, Jiangsu 221009, P.R. China
}

Received May 25, 2018; Accepted January 16, 2019

DOI: $10.3892 /$ etm.2019.7259

\begin{abstract}
Impact of hemostatic methods, electrocoagulation versus suture, on ovarian reserve and fertility in laparoscopic ovarian cystectomy was investigated. Eighty patients with bilateral ovarian cysts who underwent laparoscopic ovarian cystectomy were randomly divided into 2 groups based on the hemostatic methods: 40 in suture group and another 40 in electrocoagulation group. Blood samples were drawn from all patients at roughly three time points: Before the surgery, 1 month and 6 months after the surgery. Radioimmunoassay was performed to measure the serum levels of follicle stimulating hormone (FSH), luteinizing hormone ( $\mathrm{LH})$, estradiol (E2) and anti-Mullerian hormone (AMH). Moreover, the vaginal ultrasound examination was performed to obtain the ovarian size, peak systolic velocity (PSV) of ovarian stromal blood flow, and antral follicle count (AFC). In terms of postoperative ovarian reserve, the levels of E2 and AMH slightly decreased while the FSH level slightly increased in the suture group at both 1 and 6 months after surgery. In the electrocoagulation group, however, the levels of E2 and AMH decreased significantly while the FSH level increased significantly at 1 month after surgery. Six months after surgery, these levels all returned slightly showing some recovery of ovarian reserve. In comparison between the suture group and the electrocoagulation group, the differences in levels of E2, FSH and AMH were all statistically significant at both 1 and 6 months after surgery $(\mathrm{P}<0.05)$. Six months after surgery, the differences in AFC and PSV between the suture group and the electrocoagulation group were statistically significant $(\mathrm{P}<0.05)$. In laparoscopic ovarian cystectomy, hemostatic electrocoagulation had a more
\end{abstract}

Correspondence to: Dr Li Liang, Department of Gynecology, Xuzhou Maternity and Child Health Care Hospital, Xuzhou Medical University, 46 Heping Road, Xuzhou, Jiangsu 221009, P.R. China E-mail: pegq72@163.com

Key words: laparoscopic ovarian cystectomy, hemostatic electrocoagulation, hemostatic suture, ovarian reserve, fertility negative impact on ovarian reserve than hemostatic suture. The use of electrocoagulation for hemostasis should be minimized during the operation, and the suture method should be adopted for hemostasis and shaping of the ovarian wound.

\section{Introduction}

Benign ovarian lesions can occur at any age, which are normally treated by laparoscopic ovarian cystectomy. Laparoscopic electrocoagulation is a preferred method for hemostasis of post-cystectomy ovarian wounds because it is simple, convenient and fast. However, there is no consensus regarding the outcomes of hemostatic electrocoagulation. Several questions are being debated drawing increased attention. How does electrocoagulation for hemostasis of post-cystectomy ovarian wounds impact ovarian reserve? Does it lead to diminished ovarian function or premature ovarian failure? These are big concerns especially to those patients who still want to have children in the future $(1,2)$. In this prospective randomized controlled study, two hemostasis methods, i.e. electrocoagulation and suture, were adopted in laparoscopic ovarian cystectomy, and their impacts on ovarian reserve and fertility were evaluated.

\section{Patients and methods}

Patients. Eighty patients with bilateral ovarian cysts who were admitted to Xuzhou Maternity and Child Health Care Hospital (Xuzhou, China), from March 2010 to March 2014 were recruited into this study. The patients were randomly divided into 2 groups based on the hemostatic methods: 40 in suture group and another 40 in electrocoagulation group. The included patients all met the following criteria: $21-36$ years old; menstrual cycle 28-35 days; diagnosed with bilateral ovarian cysts by B-ultrasound; $5-8 \mathrm{~cm}$ in cyst diameter; no history of gynecological surgery in the past one year; no endocrine disorders; and no hormone use history in the past 6 months. There were no statistically significant differences in patients' preoperative general clinical records between the suture and the electrocoagulation group (Tables I and II).

The study was approved by the Ethics Committee of Xuzhou Maternity and Child Health Care Hospital, Xuzhou 
Table I. General clinical records of patients with ovarian cysts in the suture and the electrocoagulation group ( $\mathrm{n}=80$ ).

\begin{tabular}{lcccr}
\hline & \multicolumn{3}{c}{ Groups } \\
\cline { 2 - 3 } $\begin{array}{l}\text { General } \\
\text { clinical record }\end{array}$ & Suture & $\begin{array}{c}\text { Electroco- } \\
\text { agulation }\end{array}$ & t value & P-value \\
\hline Age, years & $28.59 \pm 4.22$ & $28.25 \pm 3.90$ & 0.357 & 0.797 \\
$\begin{array}{l}\text { Cyst diameter, cm } \\
\text { Left side }\end{array}$ & $5.75 \pm 0.74$ & $5.93 \pm 0.93$ & -0.858 & 0.547 \\
Right side & $5.82 \pm 0.97$ & $5.63 \pm 0.91$ & 0.86 & 0.557 \\
\hline
\end{tabular}

Medical University (Xuzhou, China). Signed informed consents were obtained from the patients or the guardians.

Surgical procedure. When induction of anesthesia was started, the patient was put in the supine position with head down and hip up. Pneumoperitoneum was established following routine procedures. The ovarian cortex was cut open over the cyst wall where the ovarian tissue was thin with few blood vessels. The incision should be away from the ovarian hilum. The cyst was stripped from the normal ovarian tissue by blunt dissection and removed by means of an endopouch. Hemostasis of the ovarian tissue was achieved by dipolar electrocoagulation in the electrocoagulation group. The ovarian wound was flushed with irrigation fluid, after which the bleeding points were identified. The bipolar electrocoagulation probe with a power setting of $25 \mathrm{~W}$ was kept in contact with the bleeding points for no more than $3 \mathrm{sec}$, generating heat and leading to hemostasis. The probe should be prevented from contacting large area of the ovarian wound. Following hemostatic electrocoagulation, the residual ovarian tissue was not sutured, and the ovarian edges were left to heal by secondary intention. Bleeding from the ovarian tissue was controlled using 4-0 absorbable sutures in the suture group. The ovarian incision was sutured in the direction perpendicular to the longitudinal axis of the ovary by taking bites from inside the ovary using a continuous suturing technique in a multipoint fashion. The suture needle should be running close to but not through the ovarian cortex, and the stitches should not be too close. All surgical procedures were successful and no severe perioperative complications occurred.

\section{Observed indicators}

Measurement of reproductive hormone levels. Venous blood samples were drawn from all patients on the 3rd day of the patient's menstrual cycle at roughly three time points: Before the surgery, 1 month and 6 months after the surgery. The serum levels of follicle stimulating hormone (FSH), luteinizing hormone (LH), estradiol (E2) and anti-Mullerian hormone $(\mathrm{AMH})$ were measured by radioimmunoassay.

Vaginal ultrasound examination. Six months after the surgery, the vaginal color Doppler ultrasonography was performed on the 5th day of the same menstrual cycle as the one when reproductive hormone levels were measured. The following indicators were obtained by the ultrasonography. First, the basal antral follicle count (AFC) in bilateral ovaries was
Table II. Types of ovarian cysts determined by postoperative pathological tests in the suture and the electrocoagulation group $(\mathrm{n}=80)$.

\begin{tabular}{lcccc}
\hline & \multicolumn{2}{c}{ Groups } & & \\
\cline { 2 - 3 } $\begin{array}{l}\text { Type of } \\
\text { ovarian cyst }\end{array}$ & Suture & $\begin{array}{c}\text { Electroco- } \\
\text { agulation }\end{array}$ & $\chi^{2}$ & P-value \\
\hline Teratoma & 13 & 16 & & \\
Endometrioma & 13 & 13 & 0.67 & 0.724 \\
Epidermoid cyst & 14 & 11 & & \\
\hline
\end{tabular}

measured, which referred to the number of antral follicles in early menstrual cycle and measuring less than $10 \mathrm{~mm}$ in diameter. Second, the peak systolic velocity (PSV) of ovarian stromal blood flow was measured. The ovarian blood flow signal was evident on the side of the ovaries. When the blood flow signal was not evident enough, the sample volume can be moved within the ovaries until a clearly visible blood flow signal appeared. The PSV was measured when the Doppler spectral waveforms became stable for consecutive five repeats. The final measured PSV of ovarian blood flow on each side was recorded as a mean value of two measurements. Third, the ovarian volume was measured as the average diameter of the largest planes at both sides.

Pregnancy during 24-month follow-up after surgery. The patients with childbearing desire were followed up for pregnancy during 24 months after surgery, in order to understand the impact of hemostatic electrocoagulation and hemostatic suture on fertility.

Criteria for ovarian reserve evaluation (3). In terms of reproductive hormone indicators, diminished ovarian reserve was defined if one of the three criteria, i.e. FSH $>15 \mathrm{U} / 1$, E2 > $293 \mathrm{pmol} / \mathrm{l}$ (or $80 \mathrm{ng} / \mathrm{l}$ ) and FSH/LH $>3.6$, was met. Normal ovarian reserve was defined if none of the above criteria was met. Premature ovarian failure was defined if FSH $>40 \mathrm{U} / 1$. In terms of vaginal ultrasound examination, the AFC in bilateral ovaries was used as a criterion. Premature ovarian failure was defined if the AFC was 0. Diminished ovarian reserve was defined if the AFC was $<5$, and normal ovarian reserve was defined if the AFC was between 5 and 15 . 
Statistical analysis. The SPSS 19.0 statistics software was used for statistical analysis. Measurement data were analyzed using the t-test, and count data using the $\chi^{2}$ test. The significance level of $\alpha=0.05$ was used. A difference was statistically significant at $\mathrm{P}<0.05$.

\section{Results}

Reproductive hormone indicators. There were no statistically significant differences in the levels of four reproductive hormones, i.e. FSH, E2, LH and AMH, before surgery between the suture and the electrocoagulation group $(\mathrm{P}>0.05)$. The levels of E2 and AMH slightly decreased while the FSH level slightly increased in the suture group at both 1 and 6 months after surgery. In the electrocoagulation group, however, the levels of E2 and AMH decreased significantly while the FSH level increased significantly at 1 month after surgery. At 6 months after surgery, these levels all returned a little bit showing some recovery of ovarian reserve. In comparison between the suture and the electrocoagulation group, the differences in levels of E2, FSH and AMH were all statistically significant at both 1 and 6 months after surgery $(\mathrm{P}<0.05)$, while the differences in LH level were not statistically significant $(\mathrm{P}>0.05)$. The detailed results are shown in Table III.

Results of vaginal ultrasound examination. There were no statistically significant differences in preoperative AFC and PSV between the suture and the electrocoagulation group. Six months after surgery, the AFC and PSV in both groups decreased, and the differences between the two groups were statistically significant $(\mathrm{P}<0.05)$. There was no statistically significant difference in the maximum diameter of the ovaries between the groups. The detailed results are shown in Tables IV and V.

Ovarian reserve. The preoperative reproductive hormone levels, antral follicle counts, and FSH/LH ratios were all within the normal range for all patients. One month after surgery, 3 patients in the electrocoagulation group had diminished ovarian reserve, but no one had premature ovarian failure. No patient had diminished ovarian reserve or premature ovarian failure in the suture group after surgery and in the electrocoagulation group at 6 months after surgery.

Postoperative menstruation and pregnancy. Menstruation basically returned to normal for all patients in 6 months after surgery. Table VI lists the postoperative pregnancy odds in 59 patients with childbearing desire after surgery. The pregnancy time was 4-24 months, averaged 10 months, after surgery. All pregnancies were normal intrauterine pregnancy, including two cases of spontaneous miscarriage. Of the 59 patients, 27 were not pregnant in the 24 -month follow-up.

\section{Discussion}

Evaluation of ovarian reserve. The ovaries are important endocrine organs for women. Diminished ovarian reserve is due to the loss of follicles and the decline in the quality of eggs. Currently, there is no existing single assay that can predict ovarian reserve with $100 \%$ accuracy. Combined evaluation 
Table IV. Results of AFC and PSV by vaginal ultrasonography before and after surgery in the two groups ( $\mathrm{n}=80)$.

\begin{tabular}{|c|c|c|c|c|c|c|c|c|}
\hline \multirow[b]{3}{*}{$\begin{array}{l}\text { Ultrasound } \\
\text { results }\end{array}$} & \multicolumn{4}{|c|}{ Before surgery } & \multicolumn{4}{|c|}{6 months after surgery } \\
\hline & \multicolumn{2}{|c|}{ Groups } & \multirow[b]{2}{*}{ t value } & \multirow[b]{2}{*}{ P-value } & \multicolumn{2}{|c|}{ Groups } & \multirow[b]{2}{*}{$\mathrm{t}$ value } & \multirow[b]{2}{*}{ P-value } \\
\hline & Suture & $\begin{array}{l}\text { Electroco- } \\
\text { agulation }\end{array}$ & & & Suture & $\begin{array}{l}\text { Electroco- } \\
\text { agulation }\end{array}$ & & \\
\hline $\mathrm{AFC}$, number & $8.450 \pm 2.218$ & $8.330 \pm 2.018$ & 2.264 & 0.793 & $7.950 \pm 2.012$ & $7.130 \pm 1.505$ & 2.076 & 0.041 \\
\hline PSV, m/s & $0.123 \pm 0.044$ & $0.121 \pm 0.041$ & 0.183 & 0.856 & $0.121 \pm 0.047$ & $0.098 \pm 0.035$ & 2.422 & 0.018 \\
\hline
\end{tabular}

Table V. Maximum ovarian diameters after surgery by vaginal ultrasonography in the two groups $(\mathrm{n}, \%)$.

\begin{tabular}{lcccc}
\hline & \multicolumn{2}{c}{ Groups } & & \\
\cline { 2 - 4 } Diameter & Suture & $\begin{array}{c}\text { Electroco- } \\
\text { agulation }\end{array}$ & $\chi^{2}$ & P-value \\
\hline$>2 \mathrm{~cm}$ & $35(87.5 \%)$ & $31(77.5 \%)$ & 1.385 & 0.239 \\
$\leq 2 \mathrm{~cm}$ & $5(12.5 \%)$ & $9(22.5 \%)$ & & \\
\hline
\end{tabular}

Table VI. Postoperative pregnancy odds of patients with childbearing desire (n, \%).

\begin{tabular}{lcccc}
\hline & \multicolumn{2}{c}{ Groups } & & \\
\cline { 2 - 4 } Pregnancy & Suture & $\begin{array}{c}\text { Electroco- } \\
\text { agulation }\end{array}$ & $\chi^{2}$ & P-value \\
\hline Yes & $17(56.7 \%)$ & $15(51.7 \%)$ & 0.290 & 0.590 \\
No & $13(43.3 \%)$ & $14(48.2 \%)$ & & \\
\hline
\end{tabular}

of multiple indicators was proved to give more accurate prediction of ovarian reserve (4). Levels of basic reproductive hormones, such as FSH, LH, E2, AMH, and parameters AFC, PSV and ovarian size measured by vaginal ultrasound exam are important indicators for evaluating ovarian reserve.

Laparoscopic ovarian cystectomy and ovarian function. Laparoscopy has become the preferred surgical method for benign ovarian tumors. Although laparoscopic ovarian cystectomy has the following advantages over laparotomy: Less invasiveness, quicker recovery, lower recurrence rate, lower postoperative adhesions, and shorter hospital time, it still causes damage to the ovaries and affects postoperative ovarian function. In China, there have been reports of postoperative premature ovarian failure (5). Therefore, in addition to preserving as much normal ovarian tissue as possible during surgery, it is also required to minimize damage in hemostatic procedure. Laparoscopic ovarian cystectomy for unilateral ovarian cyst rarely diminishes the overall ovarian function, which may be due to the compensatory function of the contralateral ovary. In this study, in order to investigate the real impact of hemostatic methods, electrocoagulation versus suture, on ovarian function, laparoscopic ovarian cystectomy was performed on patients with bilateral ovarian cysts.

In this randomized control study, two hemostatic methods, i.e. suture and electrocoagulation, were performed to manage bleeding from the ovarian wound in laparoscopic ovarian cystectomy, and ovarian reserve was evaluated after surgery. The differences in levels of E2, FSH and AMH were all statistically significant at both 1 and 6 months after surgery between the suture and the electrocoagulation group $(\mathrm{P}<0.05)$. The postoperative AFC and PSV of ovarian stromal blood flow in the electrocoagulation group were lower than those in the suture group, and the differences were statistically significant $(\mathrm{P}<0.05)$. The difference in ovarian size was not statistically significant. The findings indicated that hemostatic suture in laparoscopic ovarian cystectomy can better retain the follicles and maintain the cortical blood supply in the residual ovaries. Hemostatic electrocoagulation led to decline of ovarian function more obviously than hemostatic suture, though ovarian function can be restored to some extent after 6 months. The findings were consistent with a literature report, where it was found hemostatic suture can better maintain ovarian function than hemostatic electrocoagulation (6). Due to small sample size in the current study, the results may have some limitations.

Bipolar electrocoagulation in laparoscopic surgery may potentially cause thermal damage to the stromal blood vessels and ovarian parenchyma, thereby affecting ovarian reserve. The outcomes of bipolar electrocoagulation can be different depending on some random factors. The clinician should be skilled and be aware of key points in the procedure. The incision should be away from blood vessels while its size should be well controlled. Bipolar electrocoagulation was delivered right at the bleeding points by gently touching the points with the probe. The coagulation effect was evaluated by washing out the blood again. In this way, all the bleeding points were coagulated precisely one by one. It should be noted that the electrical power setting and the contact time during electrocoagulation must be well controlled for best outcomes. Any bleeding near the ovarian hilum should be controlled by suture if necessary, because electrocoagulation may damage the rich ovarian blood vessels in the basal area. During surgery, loss of the normal ovarian tissue attached to the wall of the cyst due to accidental excision can impact negatively on ovarian function (7). Therefore, the anatomical layers in the surgical area should be carefully distinguished in order to avoid loss of normal ovarian tissue. 
Laparoscopic ovarian cystectomy and fertility. Surgery, as well as oppression of the ovarian cysts, has an impact on ovarian reserve to a certain extent that may be different depending on the clinician's operating skills and surgical procedures (8). For patients who had combined infertility due to other reasons but still had childbearing desire, laparoscopic ovarian cystectomy was the preferred procedure, during which all factors causing infertility can be diagnosed and dealt with, such as treatment of pelvic adhesions, salpingostomy, myomectomy and adenomyomectomy. In ovarian cyst stripping, the anatomical layers should be distinguished, and as much ovarian tissue as possible should be retained, so that the ovarian function can be preserved $(9,10)$. Hemostatic suture had confirmed superiority over hemostatic electrocoagulation in terms of preserving ovarian reserve and fertility. It was also a preferred procedure for achieving reshaped ovaries after surgery. In any case, hemostatic suture was preferred to stop bleeding near the ovarian hilum if necessary. Patients with childbearing desire should be given correct and active pregnancy guidance after surgery, or use assisted reproductive technologies such as IVF.

\section{Acknowledgements}

Not applicable.

\section{Funding}

No funding was received.

\section{Availability of data and materials}

The datasets used and/or analyzed during the present study are available from the corresponding author on reasonable request.

\section{Authors' contributions}

JX designed the study, wrote the manuscript and was responsible for the statistical analysis. JX, JZ and LL were responsible for the patient treatment. HL collected the data and was responsible for follow-up. FL guided the experiments. CX was responsible for vaginal ultrasound examination. LL checked the manuscript and finalized it. All authors read and approved the final manuscript.

\section{Ethics approval and consent to participate}

The study was approved by the Ethics Committee of Xuzhou Maternity and Child Health Care Hospital, Xuzhou Medical
University (Xuzhou, China). Signed informed consents were obtained from the patients or the guardians.

\section{Patient consent for publication}

Not applicable.

\section{Competing interests}

The authors declare that they have no competing interests.

\section{References}

1. Peters A, Rindos NB and Lee T: Hemostasis during ovarian cystectomy: Systematic review of the impact of suturing versus surgical energy on ovarian function. J Minim Invasive Gynecol 24: 235-246, 2017.

2. Alammari R, Lightfoot M and Hur HC: Impact of cystectomy on ovarian reserve: Review of the literature. J Minim Invasive Gynecol 24: 247-257, 2017.

3. Kalme T, Seppälä M, Qiao Q, Koistinen R, Nissinen A, Harrela M, Loukovaara M, Leinonen P and Tuomilehto J: Sex hormone-binding globulin and insulin-like growth factor-binding protein-1 as indicators of metabolic syndrome, cardiovascular risk, and mortality in elderly men. J Clin Endocrinol Metab 90: 1550-1556, 2005.

4. Ashrafi M, Madani T, Tehranian AS and Malekzadeh F: Follicle stimulating hormone as a predictor of ovarian response in women undergoing controlled ovarian hyperstimulation for IVF. Int J Gynaecol Obstet 91: 53-57, 2005.

5. Wang H, Chen H, Qin Y, Shi Z, Zhao X, Xu J, Ma B and Chen ZJ: Risks associated with premature ovarian failure in Han Chinese women. Reprod Biomed Online 30: 401-407, 2015.

6. Pergialiotis V, Prodromidou A, Frountzas M, Bitos K, Perrea D and Doumouchtsis SK: The effect of bipolar electrocoagulation during ovarian cystectomy on ovarian reserve: A systematic review. Am J Obstet Gynecol 213: 620-628, 2015.

7. Morris RT, Gershenson DM, Silva EG, Follen M, Morris M and Wharton JT: Outcome and reproductive function after conservative surgery for borderline ovarian tumors. Obstet Gynecol 95: $541-547,2000$

8. Ding Y, Yuan Y, Ding J, Chen Y, Zhang X and Hua K: Comprehensive assessment of the impact of laparoscopic ovarian cystectomy on ovarian reserve. J Minim Invasive Gynecol 22: 1252-1259, 2015.

9. Yoon BS, Kim YS, Seong SJ, Song T, Kim ML, Kim MK and Paek JY: Impact on ovarian reserve after laparoscopic ovarian cystectomy with reduced port number: A randomized controlled trial. Eur J Obstet Gynecol Reprod Biol 176: 34-38, 2014.

10. Muzii L, Bianchi A, Crocè C, Manci N and Panici PB: Laparoscopic excision of ovarian cysts: is the stripping technique a tissue-sparing procedure? Fertil Steril 77: 609-614, 2002.

This work is licensed under a Creative Commons Attribution-NonCommercial-NoDerivatives 4.0 International (CC BY-NC-ND 4.0) License. 